\title{
A heparin binding synthetic peptide from human HIP / RPL29 fails to specifically differentiate between anticoagulantly active and inactive species of heparin David E Hoke ${ }^{1,2}$, Daniel D Carson ${ }^{3}$ and Magnus Höök*1
}

\author{
Address: ${ }^{1}$ Center for Extracellular Matrix Biology; The Texas A\&M University System Health Science Center Institute of Biosciences and Technology; \\ Houston, Texas 77030, U.S.A, ${ }^{2}$ Current Address: Department of Pathology; University of Melbourne; Parkville, Victoria 3010, Australia and \\ ${ }^{3}$ Department of Biological Sciences; University of Delaware; Newark, Delaware 19716, U.S.A \\ Email: David E Hoke - dehoke@unimelb.edu.au; Daniel D Carson - dcarson@udel.edu.au; Magnus Höök* - mhook@ibt.tamu.edu \\ * Corresponding author
}

Published: 25 February 2003

Journal of Negative Results in BioMedicine 2003, 2:1

This article is available from: http://www.jnrbm.com/content/2/I/I

(C) 2003 Hoke et al; licensee BioMed Central Ltd. This is an Open Access article: verbatim copying and redistribution of this article are permitted in all media for any purpose, provided this notice is preserved along with the article's original URL.

\begin{abstract}
Despite extensive progress in determining structures within heparin and heparan sulfate $(\mathrm{Hp} / \mathrm{HS})$ and the discovery of numerous proteinaceous binding partners for $\mathrm{HP} / \mathrm{HS}$ so far; the only detailed characterization of a specific protein-glycosaminoglycan interaction is antithrombin III (ATIII) binding to a Hp pentasaccharide containing a unique 3-O-sulfated glucosamine residue. Previously, it was reported from our laboratories that a 16 amino acid synthetic peptide derived from the $\mathrm{C}$ terminus of human HIP/RPL29 (HIP peptide-I) enriched for ATIII-dependent anticoagulant activity, presumably by specifically binding the ATIII pentasaccharide. Herein, we demonstrate that HIP peptide-I cannot enrich ATIII-dependent anticoagulant activity from a starting pool of porcine intestinal mucosa $\mathrm{Hp}$ through a bio-specific interaction. However, a HIP peptide-I column can be used to enrich for anticoagulantly active $\mathrm{Hp}$ from a diverse pool of glycosaminoglycans known as $\mathrm{Hp}$ byproducts by a mechanism of nonspecific charge interactions. Thus, HIP peptide-I cannot recognize $\mathrm{Hp}$ via bio-specific interactions but binds glycosaminoglycans by non-specific charge interactions.
\end{abstract}

\section{Introduction}

The serine protease inhibitor, antithrombin III (ATIII), is 1000 times more active when bound to a specific pentasaccharide sequence within the heparin / heparan sulfate (HS) chain [1]. While this sequence is found with a low frequency in HS, approximately $30 \%$ of the heparin molecules within a commercial preparation of porcine intestinal mucosa heparin (Hp), contains this pentasaccharide [2-5]. The ATIII - Hp complex inhibits the coagulation cascade by inactivating serine proteases, such as factor Xa (FXa) and thrombin. The interaction between ATIII and the Hp pentasaccharide (ATIII binding pen- tasaccharide) is the paradigm of a bio-specific Hp-protein interaction.

Specific protein-Hp/HS interactions involving the ATIII binding pentasaccharide or related sequences have been proposed for the fibroblast growth factor receptor (FGFR) [6], and a synthetic peptide derived from the C-terminus of human heparin/heparan sulfate interacting protein / ribosomal protein L29 (HIP peptide-1) [7]. These interactions were determined partly on the basis of column chromatography experiments. Tritiated Hp with or without unlabelled $\mathrm{Hp}$ is applied to a column of immobilized 
FGFR or HIP peptide-1 in low salt and a proportion of Hp flows through with apparently no affinity, while the remainder binds and is eluted with high salt. FGFR and HIP peptide- 1 bound fractions were enriched in ATIII-dependent anti FXa activity, presumably by specifically binding to the ATIII binding pentasaccharide or a motif associated with this sequence. The proposed bio-specific affinity of FGFR for the ATIII binding pentasaccharide or related structures has recently been challenged [8]. We also report here that HIP peptide-1 does not select for anticoagulantly active molecules in Hp or heparin byproducts through bio-specific interactions.

Previous work [7] developed five lines of evidence to indicate a specific interaction between HIP peptide- 1 and the ATIII binding pentasaccharide. Firstly, a large proportion of tritiated Hp flows through a HIP peptide- 1 column at $0.15 \mathrm{M} \mathrm{NaCl}$, suggesting that most of the molecules in a commercial preparation of tritiated Hp do not have a specific motif needed for binding. Secondly, Hp oligosaccharides generated by partial deaminative cleavage with nitrous acid show significant binding to HIP peptide-1 only when the length is an octasaccharide or higher which is similar to that seen for ATIII binding. Thirdly, tritiated Hp that binds with high affinity to HIP peptide- 1 also binds to an ATIII column with high affinity. Fourthly, Hp separated by HIP peptide-1 chromatography is enriched in ATIII-dependent FXa inhibitory activity while low affinity species show a decrease in the same activity. Lastly, HIP peptide- 1 inhibits the ability of ATIII-Hp complexes to inhibit FXa and thrombin activity, while a scrambled peptide does not. These results led us to formulate the hypothesis that HIP peptide-1 separates Hp into anticoagulantly active or inactive species by interacting with the ATIII binding pentasaccharide in a bio-specific manner.

The data presented in this paper show that fractionation of unlabelled Hp and tritiated Hp by HIP peptide- 1 display dramatic qualitative and quantitative differences. Tritiated Hp that binds to HIP peptide-1 exhibits an increase in ATIII-dependent anti FXa activity over starting material while an analogous preparation of unlabelled Hp fails to do so. The differences between HIP peptide- 1 fractionation of tritiated and unlabelled Hp is partly explained by differences in the charge profiles of these two pools as measured by anion exchange chromatographic analyses. The validity of using the commercially available tritiated $\mathrm{Hp}$ as a model for unlabelled $\mathrm{Hp}$ is discussed.

A second source of anticoagulantly active glycosaminoglycans (GAGs), called Hp byproducts, is used to analyze the binding specificity of HIP peptide-1. This is a byproduct from the preparation of commercial $\mathrm{Hp}$ and contains several GAG species, including those of the Hp/HS subclass, less sulfated than Hp [9]. Fractionation of Hp byproducts by HIP peptide- 1 chromatography yields a high affinity material that is enriched in ATIII-dependent anti FXa activity over starting material. The relative activity of the fractionated material is proportional to the salt concentration used for elution while unbound fractions are depleted in this same inhibitory activity. However, we demonstrate that this fractionation is not due to a bio-specific interaction between HIP-peptide- 1 and the ATIII binding pentasaccharide but due to a non-specific, charge based mechanism resulting in the enrichment of anticoagulantly active $\mathrm{Hp}$ from $\mathrm{Hp}$ byproducts.

\section{Results}

\section{HIP Peptide-I chromatography of heparins}

Unlabelled Hp (figure 1A) or tritiated Hp (figure 1B) was subjected to HIP peptide- 1 affinity chromatography as described in the Materials and Methods Section. Unlabelled $\mathrm{Hp}$ recovered in the $0.15 \mathrm{M} \mathrm{NaCl}$ wash corresponded to $49 \%$ of the recovered material and $51 \%$ was found in the fractions eluted with $0.50 \mathrm{M} \mathrm{NaCl}$. However, unlabelled Hp was "bleeding" from the column after extensive washing (up to 10 column volumes) with $0.15 \mathrm{M} \mathrm{NaCl}$. Conversely, tritiated Hp eluted cleanly from the column with $68 \%$ of the recovered radioactivity in the $0.15 \mathrm{M} \mathrm{NaCl}$ wash fractions and $32 \%$ in the $0.5 \mathrm{M} \mathrm{NaCl}$ eluate. The 0.5 $\mathrm{M} \mathrm{NaCl}$ eluted materials from chromatography of both Hp sources were tested in an ATIII-dependent anti FXa assay. These FXa assays (data not shown) indicated that tritiated Hp materials bound by HIP peptide-1 were enriched in anticoagulant ability over starting material while the same unlabelled Hp fraction was not. Repeated experiments where different amounts of unlabelled Hp were applied to a HIP peptide-1 column followed by analyses of bound fractions failed to show an enrichment of ATIII-dependent anti FXa ability.

\section{Anion exchange chromatography of unlabelled and tritiat- ed $\mathrm{Hp}$}

We subsequently explored the possibility that the difference in the HIP peptide-1 binding ability of unlabelled and tritiated Hp was due to a difference in the charge density of these materials. This was tested by anion exchange chromatography of unlabelled (figure 2A) or tritiated $\mathrm{Hp}$ (figure $2 \mathrm{~B}$ ) with $\mathrm{LiCl}$ gradient elution. All of the unlabelled $\mathrm{Hp}$ is found to bind to an anion exchange column and elute as a single broad peak with an average (determined from three separate experiments) peak LiCl concentration of $1.5 \mathrm{M}$. The tritiated Hp preparation is found to contain a significant amount $(38 \%)$ of material that does not bind to the anion exchange resin even before the start of the LiCl gradient. The bound fraction consists of $62 \%$ of the radioactivity and elutes at a peak $\mathrm{LiCl}$ content of $1.0 \mathrm{M}$. 

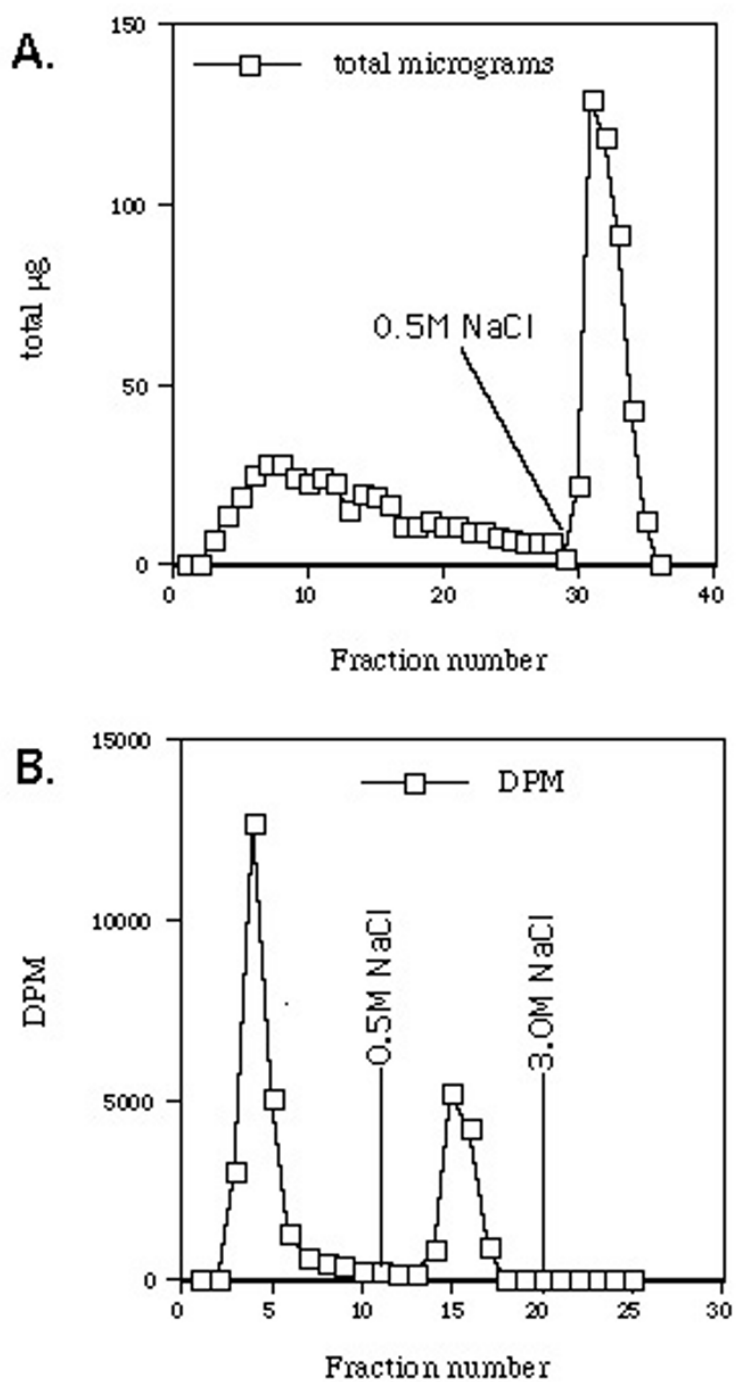

Figure I

Fractionation of Unlabelled or Tritiated Hp by HIP Peptide-I Affinity Chromatography. I mg of unlabelled $\mathrm{Hp}(\mathrm{A})$ or $40 \mathrm{ng}$ of tritiated Hp (B) was added to a HIP peptide- $I$ affinity matrix, allowed to bind for 10 min and washed with 10 column volumes of PBS before elution of bound materials with high $\mathrm{NaCl}$ as indicated. The $X$-axis corresponds to fraction number, and the $Y$-axis corresponds to total $\mu \mathrm{g}(\mathrm{A})$ or DPM (B).

\section{HIP Peptide-I affinity chromatography of heparin} byproducts

Heparin byproducts were subjected to HIP peptide-1 affinity chromatography (figure $3 \mathrm{~A}$ ). Of the material applied, $76 \%$ was recovered in flow through fractions 1-17
A.

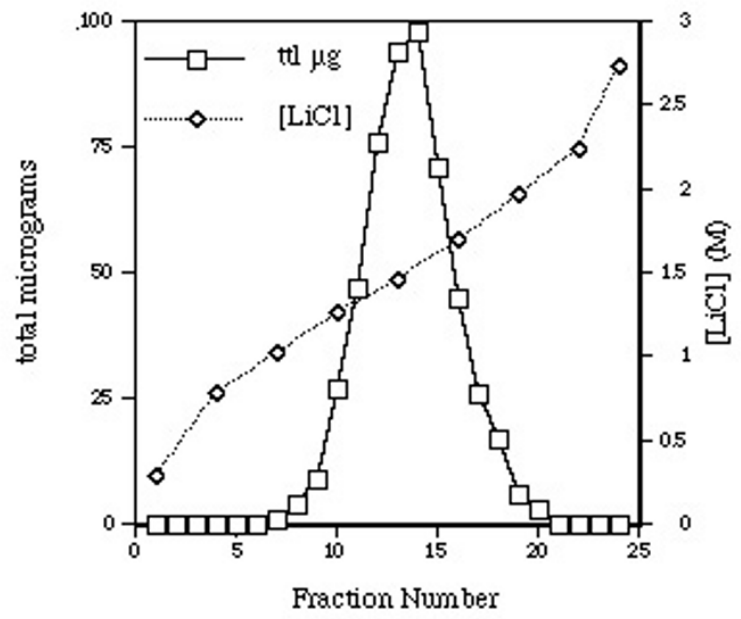

B.

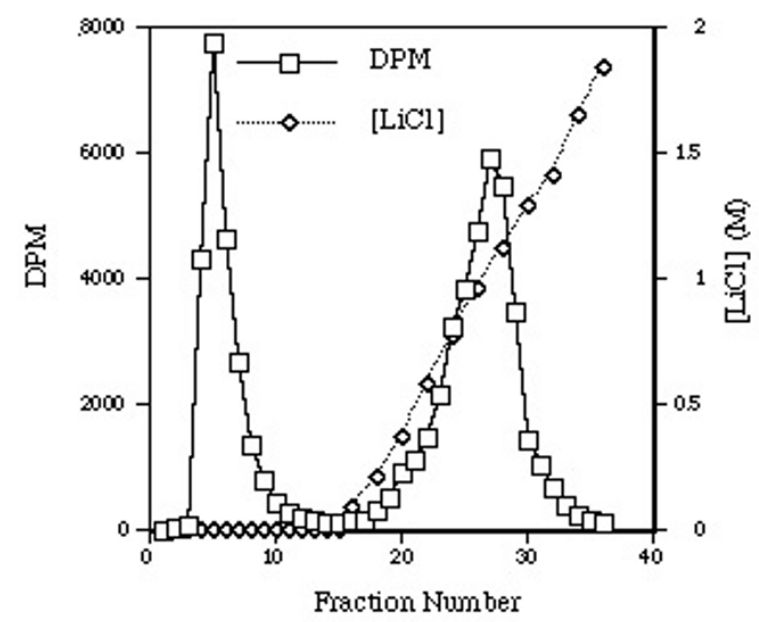

Figure 2

Anion Exchange Chromatography of Unlabelled or Tritiated Hp. Unlabelled Hp (A) or tritiated Hp (B) was added to $\mathrm{I} \mathrm{ml}$ of DEAE anion exchange resin pre-equilibrated in $0.05 \mathrm{M}$ Acetate $\mathrm{pH} 4.0$ and allowed to bind. A LiCl gradient was directly applied to the unlabelled $\mathrm{Hp}$ while the tritiated $\mathrm{Hp}$ was washed extensively in buffer without $\mathrm{LiCl}$ before the start of a $\mathrm{LiCl}$ gradient. The $\mathrm{X}$-axis corresponds to fraction number, the left $Y$-axis corresponds to total $\mu \mathrm{g}$ (A) or DPM (B), and the right $\mathrm{Y}$-axis corresponds to $\mathrm{LiCl}$ concentration.

and $24 \%$ bound to the HIP-peptide- 1 matrix and eluted between 0.22 and $0.62 \mathrm{M} \mathrm{NaCl}$, fractions $18-27$. Fractions $6,19,22$, and 24 were further analyzed for their ability to inhibit FXa activity via ATIII in an in vitro assay (figure 3B). Flow through fraction 6 exhibited a decrease in FXa inhibiting activity by $0.355 \mathrm{X}$ when compared to 


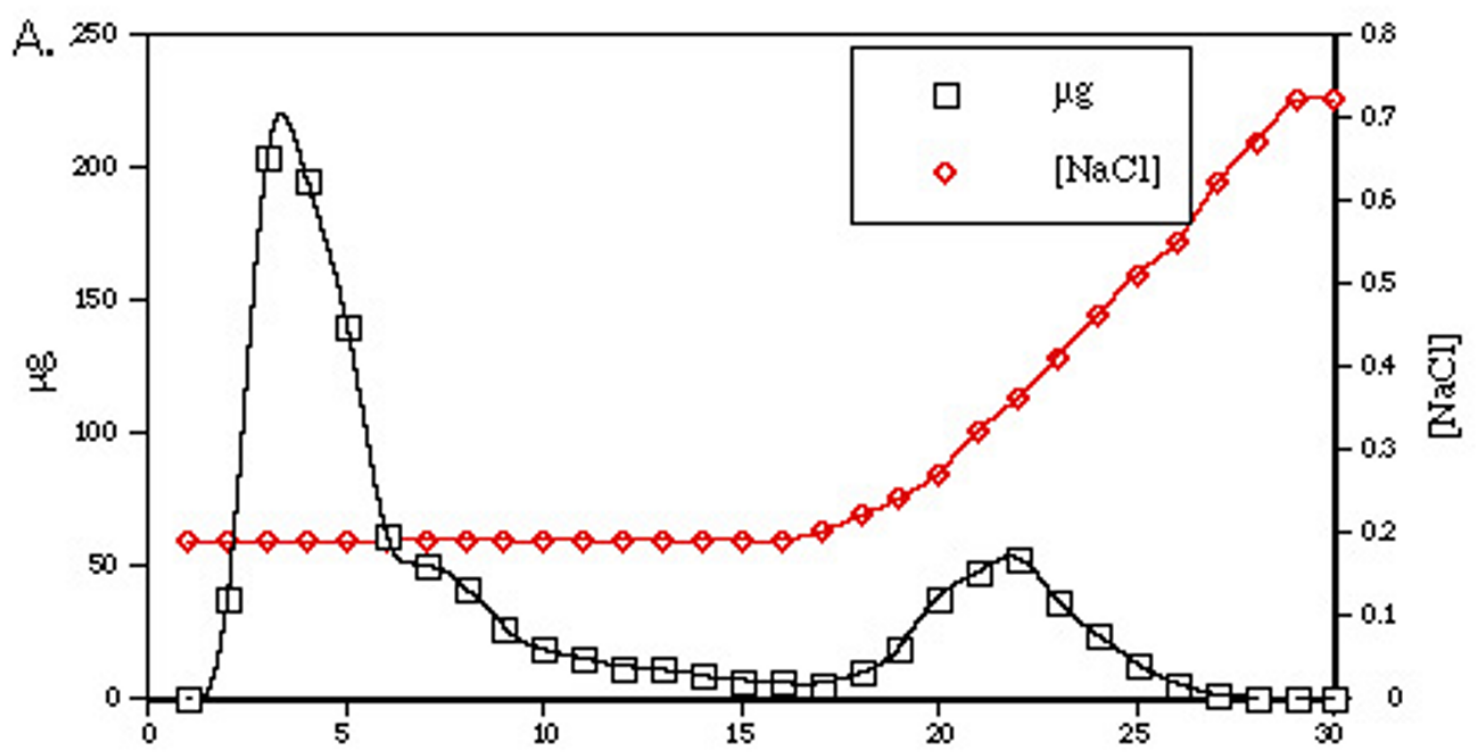

Fraction Number

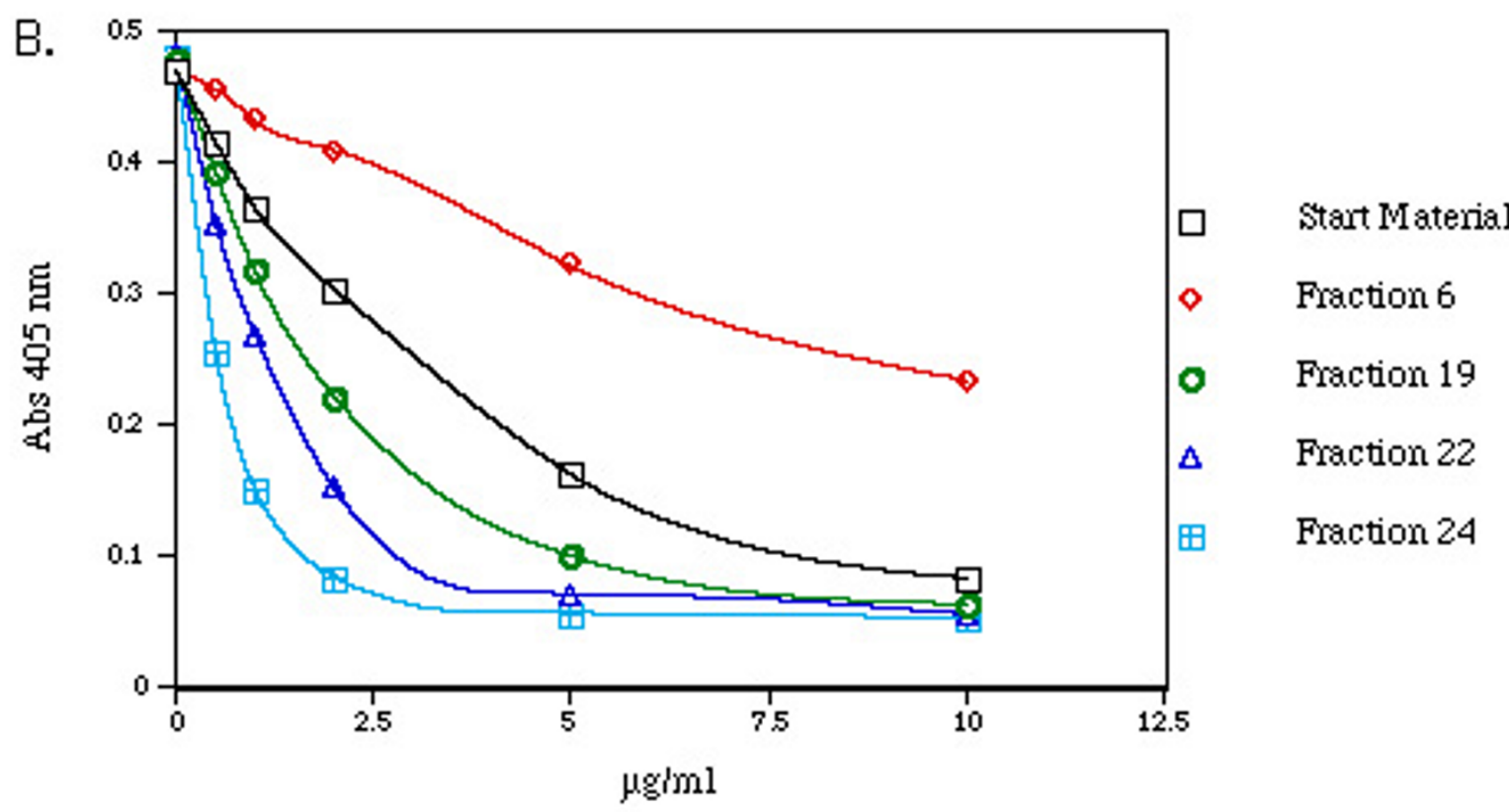

\section{Figure 3}

Fractionation of Heparin Byproducts via HIP peptide-I Chromatography and Determination of ATIII-dependent anti FXa Activity. (A) A subsaturating amount of Hp byproducts in PBS was applied to 3 mls of a HIP peptide- I affinity matrix, pre-equilibrated with PBS. Unbound materials were washed from the column by I5 ml of PBS and bound materials were eluted with a 0.15 to $0.70 \mathrm{M} \mathrm{NaCl}$ gradient. I-ml fractions were collected and the amount of material and salt content in each fraction was quantitated as in "Materials and Methods." The $X$-axis corresponds to the fraction number while the left $Y$ axis denotes the total amount of GAG material in each fraction. The right $\mathrm{Y}$-axis gives the $\mathrm{NaCl}$ concentration of each fraction. (B) Fractions 6, 19, 22, and 24 were tested in an ATIII-dependent anti FXa chromogenic assay as described in "Materials and Methods." 
starting material. All bound fractions exhibited increases in FXa inhibiting activites from 1.9X to 5.4X greater than starting material. A direct relationship between the amount of salt needed for elution from the HIP peptide-1 matrix and the fold increase in anti-FXa activity was observed.

Differential salt elution was used in HIP peptide- 1 chromatography of $\mathrm{Hp}$ byproducts to create three pools for subsequent analyses. Initially, Hp byproducts were added to HIP peptide-1 column in $0.25 \mathrm{M} \mathrm{NaCl}$ with approximately $2 \%$ bound. The $0.25 \mathrm{M} \mathrm{NaCl}$ flow through was diluted to $0.20 \mathrm{M} \mathrm{NaCl}$ and re-applied to the HIP peptide-1 column with $9.5 \%$ of the materials binding. This bound material was named HIP $0.20 \mathrm{M}$ pool. Finally, the $0.20 \mathrm{M}$ $\mathrm{NaCl}$ flow through was diluted to $0.15 \mathrm{M} \mathrm{NaCl}$ and re-applied to the column with $14 \%$ of the materials binding (HIP $0.15 \mathrm{M}$ pool) and $76 \%$ of the materials present in the final flow through (HIP flow through). As in the gradient elution of $\mathrm{Hp}$ byproducts, the materials bound in the presence of higher salt have an increase in FXa activity over starting material (data not shown). HIP $0.15 \mathrm{M}$ pool, HIP $0.20 \mathrm{M}$ pool and HIP flow through were used in subsequent analyses.

\section{Analyses of fractionated $\mathrm{Hp}$ byproducts by ion exchange chromatography}

Unfractionated Hp byproducts and fractions obtained by differential salt elution from a HIP peptide-1 column were applied to DEAE anion exchange columns and eluted with a gradient of LiCl as described in the Materials and Methods Section. No material was detected in fractions before the start of LiCl gradients. Hp byproducts displayed a very broad elution profile that is indicative of this material containing a heterogeneous population of negatively charged molecules (figure 4A). The HIP $0.20 \mathrm{M}$ pool is enriched in the most negatively charged materials whereas the HIP $0.15 \mathrm{M}$ pool appears to contain slightly less negatively charged materials which still elute late in the chromatography run compared to $\mathrm{Hp}$ byproducts starting material. Materials depleted of HIP peptide-1 binding species (HIP flow through) are completely depleted of highly negatively charged species. An ATIII affinity matrix was also used to fractionate Hp byproducts into an ATIII binding fraction with high affinity for the protease inhibitor and an ATIII non-binding fraction (see Materials and Methods section). These fractions were also analyzed for their negative charge properties by an ion exchange chromatography (figure 4B). ATIII binding species displayed an increased charge profile when compared to Hp byproducts. However, unlike the fraction depleted of HIP peptide- 1 binding material, Hp byproducts depleted of ATIII binding sites still contain highly negatively charged species when compared to the Hp byproducts starting material. Hp byproducts depleted of ATIII binding sites were also applied to a HIP peptide- 1 column and subsequently eluted with a gradient from 0.15 to $1 \mathrm{M} \mathrm{NaCl}$. It is noteworthy that significant material required greater than 0.25 $\mathrm{M} \mathrm{NaCl}$ to be eluted (data not shown) further demonstrating that ATIII and HIP peptide-1 fractionate GAGs by different types of molecular interactions.

\section{Capacity of a HIP peptide-I column for binding different GAG species}

The binding capacity of a HIP peptide- 1 column for Hp, CS-E, DS, CS-C and bovine kidney HS (BK-HS) was analyzed. Increasing concentrations of these GAGs were incubated with a defined amount of HIP peptide-1 Sepharose and the amount of GAG bound to the gel was determined (figure 5). The curves generated show binding of Hp and CS-E to HIP peptide-1 Sepharose with saturation occurring over the same range, indicative of similar binding affinities. DS showed less binding over the same range and HS or CS-C showed no binding under the experimental conditions employed. Differences in the total amounts of materials bound were noted in parallel binding experiments with $\mathrm{Hp}$ as a positive control. The total amount of CS-E and DS bound at saturation were $50 \%$ and $17 \%$, respectively, of the total amount of $\mathrm{Hp}$ bound at saturation. These results are consistent with the hypothesis that the HIP peptide-1 / GAG interaction depends solely on nonspecific charge interactions.

\section{Discussion}

Previous work [7] suggested that HIP peptide-1 could enrich for anticoagulantly active Hp on the basis of a biospecific interaction with the ATIII binding pentasaccharide. The goal of the current study was to substantiate this hypothesis. However, an overwhelming amount of data presented here demonstrates that the underlying hypothesis is invalid. Although we can enrich for anticoagulantly active Hp by HIP peptide- 1 affinity chromatography using tritiated heparin or Hp byproducts as a starting material, this enrichment is not due to bio-specific interactions. Furthermore, repeated chromatography experiments with unlabelled conventional $\mathrm{Hp}$ as the starting material failed to significantly enrich for ATIII binding Hp. Lastly, Zhang et al. [10] have recently reported that HIP peptide-1 does not protect anticoagulantly active HS from complete heparitinase digestion.

It is clear from the current data that our samples of unlabelled and tritiated $\mathrm{Hp}$ are not biochemically similar. A significant proportion (38\%) of tritiated $\mathrm{Hp}$ does not bind an anion exchange column while $100 \%$ of unlabelled Hp does. We hypothesize that this sample of tritiated $\mathrm{Hp}$ contains $38 \%$ free label and thus has no apparent negative charge. Since the fractionation of this particular preparation of tritiated Hp over HIP peptide- 1 is similar to the previously published observations, we can only 


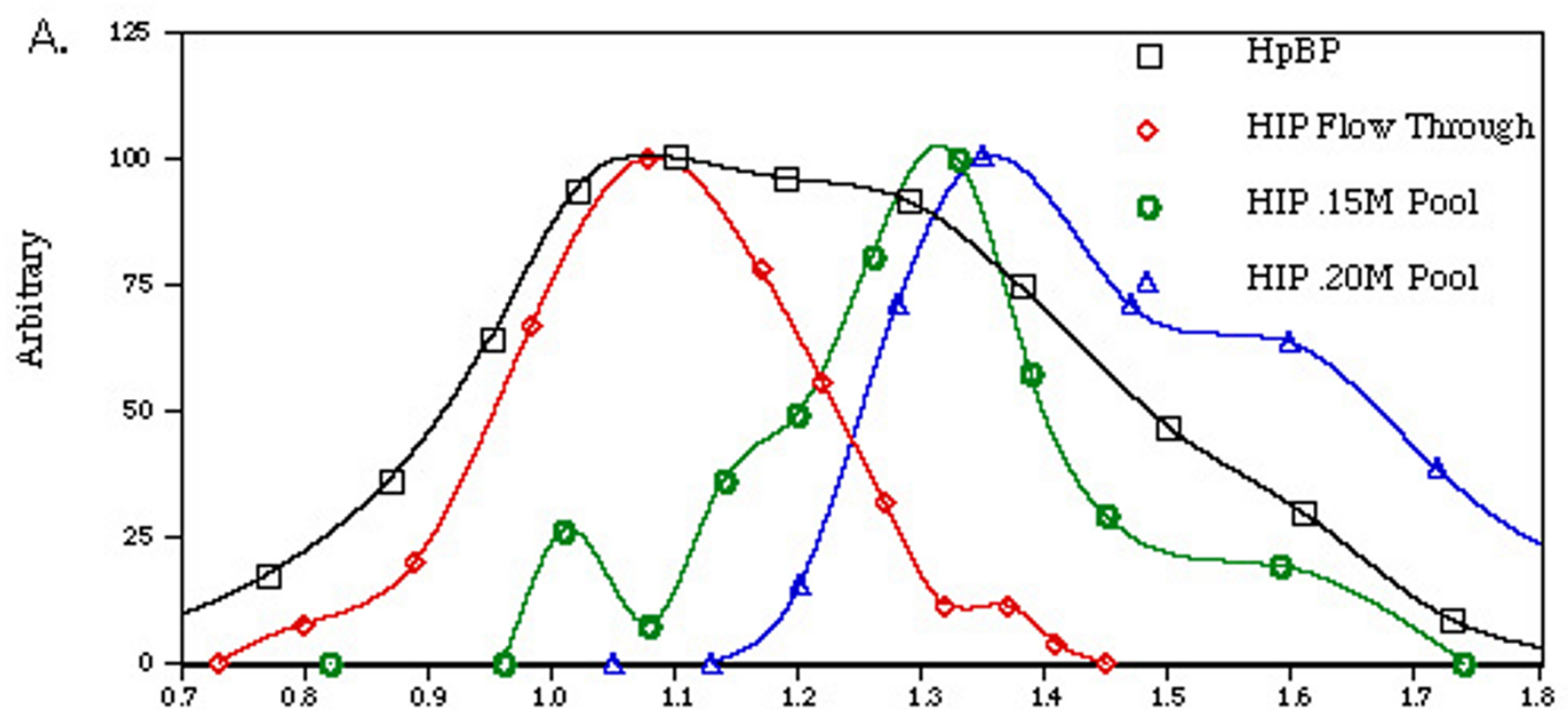

[LiCl]

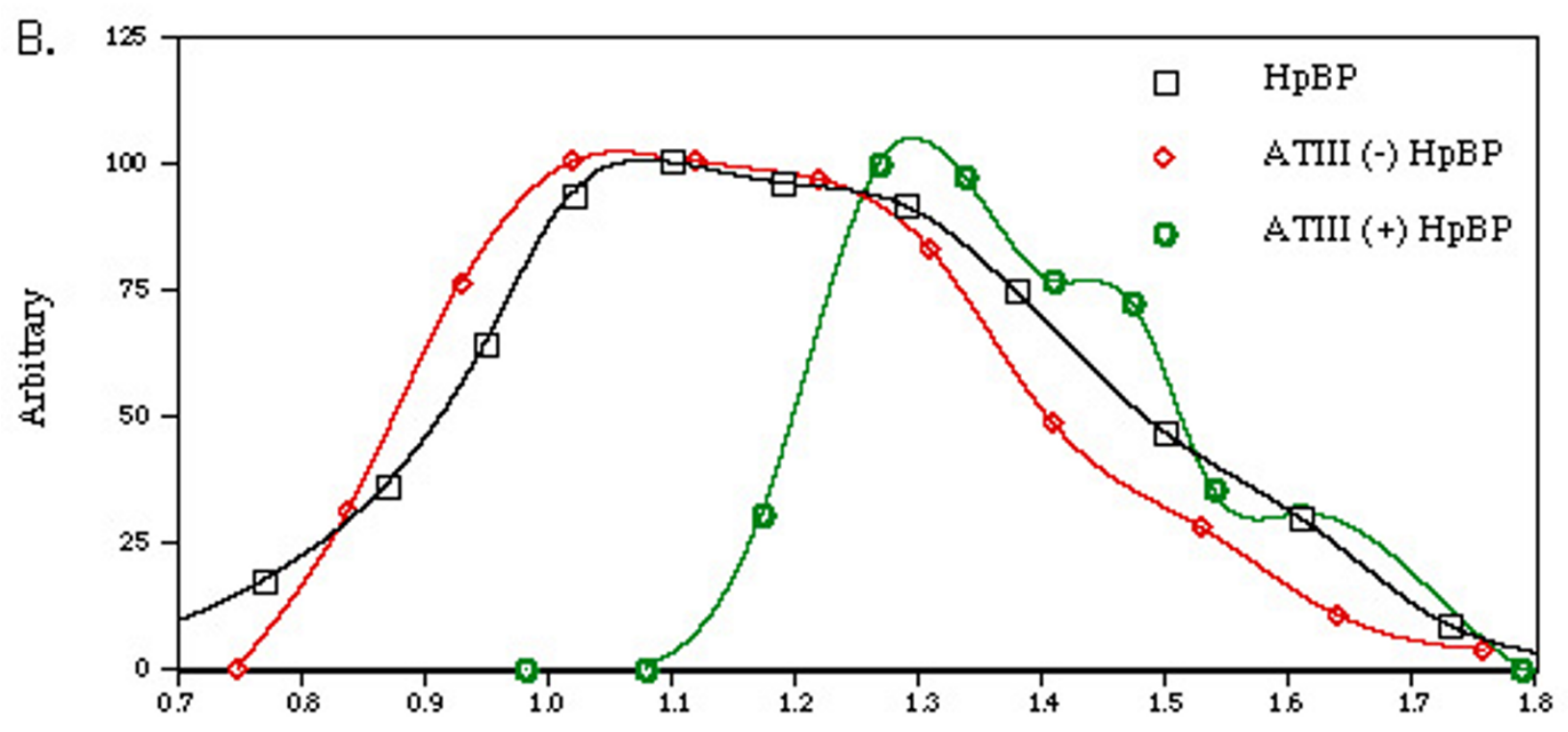

[LiCl]

Figure 4

Elution Profiles of Hp Byproducts or Fractionated Pools from Anion Exchange Chromatography with Gradient Elution. Hp byproducts starting material $(\mathrm{HpBP})$ or materials from fractionation via HIP peptide-I (A) or ATIII (B) were applied to a I-ml bed volume of DEAE sepharose in $.05 \mathrm{M}$ acetate, $\mathrm{pH} 4.0$. Columns were washed with 10 column volumes of acetate buffer before application of $\mathrm{LiCl}$ gradients. All material bound to DEAE column and $100 \%$ eluted with $\mathrm{LiCl}$. One-ml fractions were collected and analyzed for GAG and $\mathrm{LiCl}$ content as in "Materials and Methods." Arbitrary values of $\mathrm{I00}$ were set for the elution peaks for each of the column runs with every other point multiplied by the same factor. The resulting plots are of $\mathrm{LiCl}$ concentration (X-axis) versus arbitrary amount of GAG (Y-axis). 


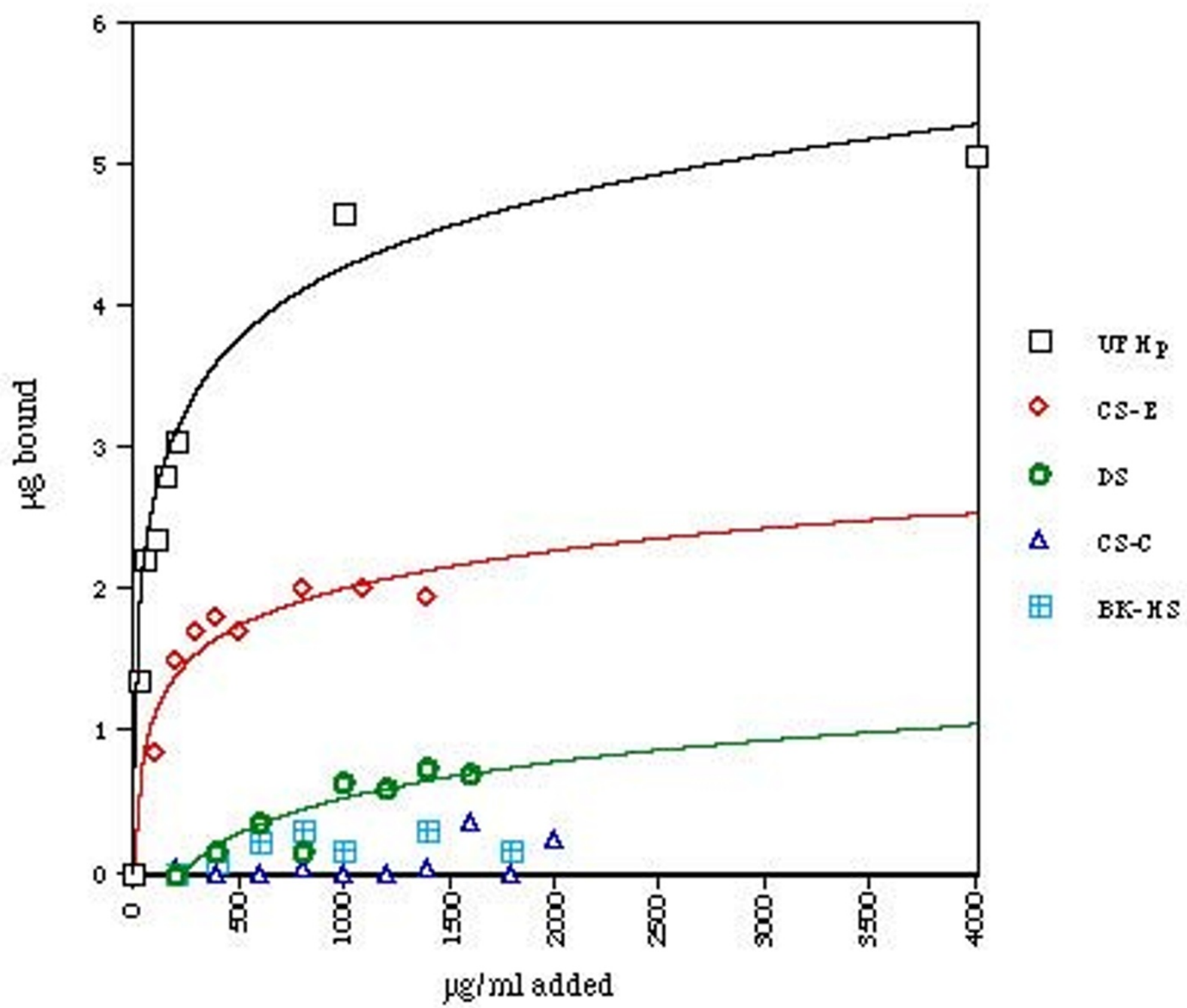

Figure 5

Binding Curves of GAGs to HIP Peptide- I. Dilutions of Hp, CS-E, DS, CS-C or Bovine kidney-HS (BK-HS) were added in $400 \mu \mathrm{l} \mathrm{PBS}$ to $100 \mu \mathrm{l}$ of a I:I slurry of HIP peptide-I sepharose : PBS. After a I0-minute incubation, the tubes were washed with three serial dilutions of $500 \mu$ PBS. Bound materials were eluted with $2 \mathrm{M} \mathrm{NaCl}$ and quantified as in "Materials and Methods." Values shown are the total amounts bound (Y-axis) versus the concentration of the material added (X-axis). Hp, CS-E, and DS were fitted to a logarithmic curve with $r$ squared values of 0.81 or greater.

conclude that the initial preparation also contained significant amounts of "free label". The presence of "free label" would explain the repeated enrichment of ATIII-dependent anti FXa ability seen in tritiated Hp. Rather than enriching for the ATIII binding pentasaccharide by HIP peptide-1 chromatography of tritiated $\mathrm{Hp}$, this chroma- tography enriches for Hp, leaving behind "free label". This would result in an apparent enrichment of activity per DPM in bound tritiated Hp materials and a depletion of activity per DPM in unbound tritiated Hp materials. 
When the starting material used for fractionation on a HIP peptide- 1 column is Hp byproducts, a charge dependent increase in ATIII-dependent anti FXa activity for bound materials is seen. Hp byproducts are a very heterogeneous mixture of GAGs. Due to the nature of the Hp/HS biosynthetic pathways, the ATIII binding pentasaccharide is formed preferentially in highly negatively charged regions [1]. Our hypothesis is that HIP peptide-1 chromatography does not enrich for anticoagulant activity from Hp byproducts on the basis of a bio-specific interaction with the ATIII binding pentasaccharide but rather due to non-specific charge interactions. This hypothesis was substantiated by comparing the charge characteristics of $\mathrm{Hp}$ byproducts depleted of HIP peptide- 1 or ATIII binding activity. Also, the binding ability of HIP peptide- 1 for Hp byproducts depleted of ATIII binding activity was determined. Firstly, elution profiles from anion exchange chromatography show that ATIII binding species have an increased charge profile when compared to starting material, but the ATIII-depleted materials are only slightly shifted to a less negatively charged profile compared to starting material. In contrast, HIP peptide- 1 depleted Hp byproducts are essentially depleted of its most negatively charged species. This shows that highly negatively charged species within Hp byproducts are associated with, but not sufficient for ATIII interaction while HIP peptide- 1 has a simple charge requirement. Secondly, HIP peptide-1 binds some ATIII depleted Hp byproducts indicating that the ATIII binding pentasaccharide is not essential for HIP peptide-1 binding. The inherently high negative charge of ATIII binding species $[11,12]$ and the charge heterogeneity of Hp byproducts makes the HIP peptide- 1 separation of Hp byproducts into pools with low and high anticoagulant activity possible. In contrast, the relatively homogeneously charged Hp preparations tested are refractory to a similar separation.

Binding studies suggest that HIP peptide- 1 has a selectivity for GAGs of the Hp/HS subclass [13]. This is demonstrated by the inability of CS-A, DS, CS-C, KS, and HA to act as effective competitors for HIP peptide-1 binding to tritiated Hp. Additionally, HIP peptide-1 is found to bind subsets of cell surface JAR cell HS and DS, suggesting differentiation within a class of GAG. Binding potential is increased in pools of JAR cell HS or DS that have longer length and higher sulfation content. Likewise, Hp byproducts bound in the presence of $0.20 \mathrm{M}$ have increased size (D.E.H. and M.H. unpublished observations) and charge when compared to Hp byproducts bound in $0.15 \mathrm{M} \mathrm{NaCl}$. The new interpretation of this data is that HIP peptide- 1 binds GAGs via a threshold charge and not due to inherent bio-specificity. This is further supported by the determined hierarchy of binding potential; Hp > CS-E > DS > CS-C = BK-HS, which mimics the charge density order of the GAGs; suggesting that the sulfation content is the most important factor in the interaction with HIP peptide- 1 and not the subclass of GAG.

Theoretically, it should be possible to create linear peptides that can specifically bind to 'sequences' within the linear GAG chain. Our current knowledge on $\mathrm{Hp} / \mathrm{HS}$ binding motifs has come from an examination of $\mathrm{Hp} / \mathrm{HS}$ binding proteins that identified the XBBXBX and XBBBXXBX motifs where $\mathrm{X}$ is an uncharged or hydrophobic amino acid and B is a basic amino acid [14]. It is important to note that HIP peptide-1 (CRPKAKAKAKAKDQTK) does not exactly correspond to either of these motifs yet binds Hp/HS. However, studies have shown that concatamers of peptides that conform to these consensus motifs have binding affinity proportional to the number of subunits [15] and can reverse the antithrombotic activity of $\mathrm{Hp}$ in vivo [16]. A natural example of a concatamer of Hp/HS binding motifs is human HIP/ RPL29. This protein is highly basic with $29.5 \%$ of Lys/Arg content distributed evenly throughout the protein. Studies with deletion mutants of human HIP/RPL29 show that $\mathrm{Hp} / \mathrm{HS}$ binding ability increases with the length of deletion mutant, irrespective of domain [17]. Concatamers of $\mathrm{Hp} / \mathrm{HS}$ binding sequences may be a common mechanism of protein/GAG interactions. Future work will be aimed at identifying novel proteins and peptide sequences that specifically interact with ATIII binding Hp/HS.

\section{Materials and Methods \\ Materials}

Porcine intestinal mucosa heparin (product number H3393), bovine kidney heparan sulfate, dermatan sulfate, and chondroitin sulfate C, were purchased from Sigma. Chondroitin Sulfate E was purchased from CalBiochem. Tritiated heparin $(0.44 \mathrm{mCi} / \mathrm{mg})$ was purchased from NEN life science products. DEAE resin was purchased from Pharmacia. Heparin byproducts were obtained from Scientific Protein Laboratories (division of Viobin corporation) Waunakee, WI. Human blood plasma was obtained from the Houston Blood Center (Houston, TX).

\section{HIP peptide-I affinity chromatography}

HIP peptide-1 affinity resin was prepared as in Liu et al., [7]. One mg Hp or $40 \mathrm{ng}$ tritiated Hp was applied to HIP peptide- 1 affinity resin in $0.15 \mathrm{M} \mathrm{NaCl}$, phosphate buffer, allowed to bind for $10 \mathrm{~min}$ and washed with 10 column volumes of $0.15 \mathrm{M} \mathrm{NaCl}$ phosphate buffer. Then $0.5 \mathrm{M}$ $\mathrm{NaCl}$ was used to elute any bound $\mathrm{Hp}$ or tritiated Hp. A final elution with $3 \mathrm{M} \mathrm{NaCl}$ was employed for tritiated Hp. Hp byproducts were applied to 3 mls of a HIP peptide-1 affinity matrix in PBS, washed with $15 \mathrm{mls}$ PBS, and eluted w/ a $0.15 \mathrm{M}$ to $0.70 \mathrm{M} \mathrm{NaCl}$ gradient. Material for the bulk separation of $\mathrm{Hp}$ byproducts were initially added in $0.25 \mathrm{M} \mathrm{NaCl}$, under subsaturating conditions, washed with ten column volumes of $0.25 \mathrm{M} \mathrm{NaCl}$ and finally 
eluted with $1 \mathrm{M} \mathrm{NaCl}$. Subsequent experiments for the bulk separation of Hp byproducts took the flow through from the previous salt separation and diluted them to 0.2 $\mathrm{M}$, and then $0.15 \mathrm{M}$ for the serial separation of materials with decreasing stringency. Five different column runs were done at each step to create HIP $0.20 \mathrm{M}$, HIP $0.15 \mathrm{M}$ and HIP flow through pools. One $\mathrm{ml}$ fractions were collected and salt concentrations were determined against a standard curve using a conductivity meter.

\section{GAG quantitation}

A Blyscan (Biocolor, Ltd.; Belfast, Ireland) assay was used for the quantitation of GAGs in experiments using unlabelled GAGs. The assay is based on the specific binding of the cationic dye; 1,9-dimethyl methylene blue to sulphated GAGs [18]. A standard curve of the relevant material was made during each quantitation that had a correlation coefficient of 0.96 or greater. Unknown solutions were diluted to $<0.5 \mathrm{M} \mathrm{NaCl}$ where applicable and quantitation performed in at least duplicate. Quantitation of tritiated Hp was performed by liquid scintillation counting.

\section{Anion exchange chromatography for the determination of GAG charge}

A $1 \mathrm{ml}$ DEAE column was pre-equilibrated in $0.05 \mathrm{M}$ Acetate $\mathrm{pH} 4.0$ and GAGs applied [19]. Columns were washed in acetate buffer for 10 column volumes before the start of $\mathrm{LiCl}$ gradients ranging from 0 to $2 \mathrm{M} \mathrm{LiCl}$. In some experiments where it was previously determined that $100 \%$ of the GAGs bound to the column, washing steps were omitted and $\mathrm{LiCl}$ gradients started at fraction one. One-ml fractions were collected and quantified in the Blyscan assay against a standard curve or radioactivity counted by liquid scintillation counting. A standard curve for LiCl was also made and read on a conductivity meter enabling the conversion of experimental conductivity readings to $\mathrm{LiCl}$ concentration. Data from these experiments were analyzed by assigning the maximum peak of elution an arbitrary value of 100 . All other values were multiplied by the same factor and elution profiles from $\mathrm{LiCl}$ concentration (X-axis) versus arbitrary (Y-axis) were made. A line running through the transformed elution profiles 50 arbitrary value was made and the two intersection points of $\mathrm{LiCl}$ concentration were noted. These two intersection values were averaged to determine the $\mathrm{LiCl}$ concentration for elution peaks. The $\mathrm{LiCl}$ concentration for elution peak in experiments where multiple elution profiles were made, was an average of the individual averages.

\section{FXa activity of GAG fractions}

Clinical kits (Sigma, St. Louis, MO) were used in the determination of ATIII - dependent Hp activity in FXa inhibition as described in the corresponding instructions except that Hp / Hp byproducts materials were added in place of plasma. Briefly, this kit uses ATIII, FXa, and a chromogenic substrate for FXa to measure Hp concentrations in blood. This assay has been utilized to measure the relative activity of fractionated $\mathrm{Hp}$ byproducts to accelerate the inactivation of FXa by ATIII-Hp complexes as measured by comparisons in ATIII-GAG - dependent inhibition of substrate cleavage measured at $405 \mathrm{~nm}$. Glycosaminoglycan containing solutions were diluted to 0.15 $\mathrm{M} \mathrm{NaCl}$ before use in the assay. Enrichment or depletion of FXa activity was determined by identifying the concentration of Hp byproducts at which FXa activity was inhibited $1 / 2$ maximally $\left(1 / 2 \max _{i n h}\right)$. The $1 / 2 \max _{i n h}$ concentration of the starting material was divided by the $1 / 2$ max $_{\text {inh }}$ concentration of the sample to determine fold increase in FXa activity over starting material.

\section{Antithrombin III affinity chromatography}

Antithrombin III was purified from human plasma as described in Wickerhauser and Williams [20]. The purified ATIII was then linked to CNBr activated Sepahrose in the presence of excess $\mathrm{N}$-acetylated $\mathrm{Hp}$ as in Höök et al., [2]. Hp byproducts were applied to the ATIII column in PBS, washed with 10 column volumes and bound material eluted in phosphate buffer containing $3 \mathrm{M} \mathrm{NaCl}$. Flow through materials was subjected to ATIII chromatography 5 times. After the third time, no bound material was detected. Thus, th 5 times ATIII flow through is completely depleted of ATIII binding sites. This was confirmed by a lack of activity in the FXa assay (data not shown).

\section{GAG binding assays}

Multiple tubes containing $100 \mu \mathrm{l}$ of a 1:1 suspension of HIP peptide-1 Sepharose in PBS were assembled. Four hundred $\mu$ l of UF Hp (Sigma), bovine kidney heparan sulfate (Sigma), chondroitin sulfate-C (Sigma), dermatan sulfate (Sigma), or chondroitin sulfate-E (CalBiochem), was added in a range of concentrations from $50 \mu \mathrm{g} / \mathrm{ml}$ to $1500 \mu \mathrm{g} / \mathrm{ml}$. The mixtures were incubated at room temperature for thirty minutes after an initial vortexing. The tubes were then centrifuged at $16,000 \times \mathrm{G}$ for $5 \mathrm{~min}$ and the liquid aspirated, leaving resin in the tube. Then, 500 $\mu \mathrm{l}$ of PBS was added to the resin bed, vortexed, centrifuged, and liquid aspirated. This cycle was done three times to ensure a 1:1000 final dilution of initially added GAGs. After a final aspiration, $50 \mu \mathrm{l}$ of $2 \mathrm{M} \mathrm{NaCl}$ in phosphate buffer was added, releasing any bound material into the liquid phase. Aliquots of this material were quantified by the Blyscan assay and $\mu \mathrm{g} / \mathrm{ml} \mathrm{GAG} \mathrm{added} \mathrm{(X-axis)} \mathrm{versus}$ total $\mu$ g GAG bound (Y-axis) plots were made.

\section{Abbreviations}

heparin / heparin from porcine intestinal mucosa - Hp; factor Xa - FXa; antithrombin III - ATIII; heparan sulfate - HS; chondroitin sulfate - CS, dermatan sulfate - DS, heparin/heparan sulfate interacting protein / ribosomal 
protein L29 - HIP/RPL29; heparin/heparan sulfate interacting protein / ribosomal protein L29 peptide-1 - HIP peptide-1; glycosaminoglycan - GAG; phosphate buffered saline - PBS

\section{Acknowledgements}

We would like to thank Dr. Patrick N. Shaklee from Scientific Protein Laboratories for samples of $\mathrm{Hp}$ byproducts. This work was supported by an Advanced Research Proposal grant from the Texas Coordinating Board (Grant 000089-0021-1999).

\section{References}

I. Salmivirta $M$, Lidholt $K$ and Lindahl $U$ Heparan sulfate: a piece of information. FASEB / 1996, I0:1270-9

2. Hook M, Bjork I, Hopwood JJ and Lindahl U Anticoagulant activity of heparin: separation of high activity and low activity heparin species by affinity chromatography on immobilized antithrombin. FEBS Lett 1976, 66:90-93

3. Lam LH, Silbert JE and Rosenberg RD The separation of active and inactive forms of heparin. Biochem Biophys Res Commun 1976, 69:570-577

4. Lindahl U, Backstrom G, Hook M, Thunberg L, Fransson L-A and Linker A Structure of the antithrombin-binding site in heparin. Proc Natl Acad Sci USA 1979, 76:3198-3202

5. Lindahl U, Backstrom G, Thunberg L and Leder IG Evidence of a 3O-sulfated D-glucosamine residue in the antithrombin-binding sequence of heparin . Proc Natl Acad Sci USA 1980, 77:655I6555

6. McKeehan WL, Wu X and Kan M Requirement for anticoagulant heparan sulfate in the fibroblast growth factor receptor complex. J Biol Chem 1999, 274:215II-4

7. Liu S, Zhou F, Hook M and Carson DD A heparin-binding synthetic peptide of heparin/heparan sulfate-interacting protein modulates blood coagulation activities. Proc Natl Acad Sci U S A 1997, 94: 1739-44

8. Loo BM, Kreuger J, Jalkanen M, Lindahl $U$ and Salmivirta M Binding of heparin/heparan sulfate to fibroblast growth factor receptor. J Biol Chem 2001, 276: I6868-76

9. Conrad HE Heparin binding proteins New York-London Academic Press 1998,

10. Zhang L, Beeler DL, Lawrence R, Lech M, Liu J, Davis JC, Shriver Z, Sasisekharan R and Rosenberg RD 6-O-Sulfotransferase-I represents a critical enzyme in the anticoagulant heparan sulfate biosynthetic pathway. J Biol Chem 200I, 276:423 II-2I

I I. Hurst RE, Menter JM, West SS, Settine JM and Coyne EH Structural basis for the anticoagulant activity of heparin. Relationship to the number of charged groups. Biochemistry 1979, I 8:4283-7

12. Hurst RE, Poon MC and Griffith MJ Structure-activity relationships of heparin. Independence of heparin charge density and antithrombin-binding domains in thrombin inhibition by antithrombin and heparin cofactor II. J Clin Invest 1983, 72: I042-5

13. Liu S, Julian J and Carson DD A peptide sequence of heparin/ heparan sulfate (HP/HS)-interacting protein supports selective, high affinity binding of HP/HS and cell attachment. J Biol Chem 1998, 273:9718-26

14. Cardin AD and Weintraub HJ Molecular modeling of proteinglycosaminoglycan interactions. Arteriosclerosis 1989, 9:21-32

15. Verrecchio A, Germann MW, Schick BP, Kung B, Twardowski T and San Antonio JD Design of peptides with high affinities for heparin and endothelial cell proteoglycans. J Biol Chem 2000, | 7:770|-7

16. Schick BP, Gradowski JF, San Antonio JD and Martinez J Novel design of peptides to reverse the anticoagulant activities of heparin and other glycosaminoglycans. Thromb Haemost 200I, 85:482-7

17. Hoke DE, LaBrenz SR, Hook M and Carson DD Multiple domains contribute to heparin/heparan sulfate binding by human HIP/L29. Biochemistry 2000, 39:15686-94

18. Farndale RW, Sayers CA and Barrett AJ A direct spectrophotometric microassay for sulfated glycosaminoglycans in cartilage cultures. Connect Tissue Res 1982, 9:247-248

19. Forsberg E, Pejler G, Ringvall M, Lunderius C, Tomasini-Johansson B, Kusche-Gullberg M, Eriksson I, Ledin J, Hellman L and Kjellen L Ab- normal mast cells in mice deficient in a heparin-synthesizing enzyme. Nature 1999, 19:773-6

20. Wickerhauser $M$ and Williams $C$ A single-step method for the isolation of antithrombin III. Vox Sang 1984, 47:397-405
Publish with Bio Med Central and every scientist can read your work free of charge

"BioMed Central will be the most significant development for disseminating the results of biomedical research in our lifetime. "

Sir Paul Nurse, Cancer Research UK

Your research papers will be:

- available free of charge to the entire biomedical community

- peer reviewed and published immediately upon acceptance

- cited in PubMed and archived on PubMed Central

- yours - you keep the copyright

Submit your manuscript here:

http://www.biomedcentral.com/info/publishing_adv.asp
BioMedcentral 